\title{
Comparison of Conventional and Asymmetric Aircraft Configurations using CEASIOM
}

\author{
Cormac McFarlane \&* Thomas S. Richardson, ${ }^{\dagger}$ \\ Department of Aerospace Engineering, University of Bristol, \\ Queens Building, University Walk, Bristol, BS8 1TR, UK \\ Andrea Da Ronch \& Ken Badcock \\ School of Engineering, University of Liverpool, \\ Liverpool, L69 3BX, UK
}

\begin{abstract}
This paper investigates whether drag reductions in cruise can be achieved for an aircraft by selecting a three-lifting-surface asymmetric design. A conventional T-tail design based on the existing EA500 Very Light Jet was selected as the baseline aircraft, and this was redesigned into a novel asymmetric configuration. CEASIOM, the Computerized Environment for Aircraft Synthesis and Integrated Optimization Methods, was then used to generate aerodynamic data sets for both aircraft, trim them at the cruise condition, and compare the thrust required to achieve trim.
\end{abstract}

\section{Acronyms}

CEASIOM Computerized Environment for Aircraft Synthesis and Integrated Optimization Methods

CG Center of Gravity

GUI Graphical User Interface

MAC Mean Aerodynamic Chord

SCAA Stability and Control Analyser and Assessor

\section{Introduction}

In this paper the Computerized Environment for Aircraft Synthesis and Integrated Optimization Methods (CEASIOM) is used to assess whether the suggested drag reduction of using an asymmetric three-liftingsurface aircraft design can be achieved in cruise.

CEASIOM is currently being developed within the frame of the SimSAC Project (Simulating Aircraft Stability And Control Characteristics for Use in Conceptual Design) sponsored by the European Commission 6th Framework Programme. It uses adaptive-fidelity CFD to create aerodynamic datasets for aircraft, after which trim points can be calculated for the aircraft, and stability and control characteristics can be analysed using integrated software tools. Details of CEASIOM and its tools are given in Section II.

A baseline configuration was selected against which the novel design could be compared with CEASIOM being used to analyse both aircraft. The novel configuration was designed to fulfill the same role as the baseline aircraft, with design decisions made to ensure that any drag reductions were a result of using three lifting surfaces rather than from relaxing requirements (such as the static margin). When generating the aerodynamic data sets for both aircraft the same methods were used, again to ensure the comparison was as accurate as possible. Details of the two aircraft configurations are given in Section III.

The aerodynamic data sets generated for both aircraft were then used by CEASIOM to trim both aircraft in the cruise configuration. This enabled the thrust required to achieve trim in cruise to be compared for

\footnotetext{
${ }^{*}$ Research Assistant, C.McFarlane@bristol.ac.uk

${ }^{\dagger}$ Lecturer in Flight Mechanics, Member AIAA, Thomas.Richardson@bristol.ac.uk
} 
the two aicraft to determine whether the novel design was more efficient. A comparison of the two aircraft trimmed for cruise is given in Section IV.

\section{CEASIOM}

The Computerized Environment for Aircraft Synthesis and Integrated Optimization Methods (CEASIOM) application is a conceptual aircraft design tool that enhances the process of conceptual design by incorporating stability and control assessment at an early stage. CEASIOM consists of several modules, with those which are used for the work in this paper described below. Further details on CEASIOM can be found in References. ${ }^{1,2}$

\section{II.A. AcBuilder}

The AcBuilder module of CEASIOM is used to define a simplified aircraft geometry using around 100 parameters. Examples of such parameters include the wing span, wing area, leading edge sweep etc. A visual representation of the aircraft is generated by the software, as shown in Figure 1, allowing the user to verify the impact of changing any parameter on the aircraft model.

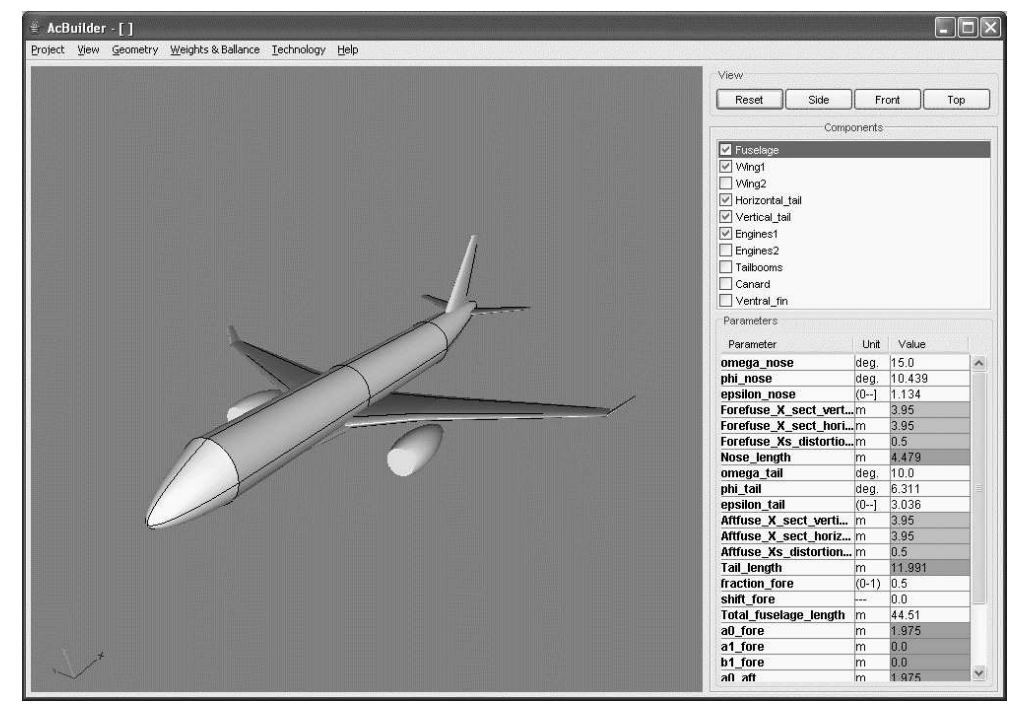

Figure 1. AcBuilder GUI

This module also estimates weights and balance parameters for the concept aircraft, which are necessary for assessing its stability and control characteristics.

\section{II.B. AMB}

The Aerodynamic Model Builder (AMB) module of CEASIOM generates aerodynamic data sets for the aircraft geometry defined in AcBuilder using variable fidelity aerodynamic predictions codes including:

- DATCOM - A handbook method based on empirical data

- Tornado - A vortex-lattice method for low-speed aerodynamics and aeroelasticity

- Edge - An inviscid Euler solver for high-speed aerodynamics (based on meshes generated by SUMO)

These codes allow aerodynamic coefficients to be generated for steady, dynamic and unsteady conditions. In addition to the user selecting the appropriate prediction code they also control the number of data points for each of the tables. Tables can therefore be tailored to suit the needs of the user. Figure 2 shows the GUI for $\mathrm{AMB}$. 


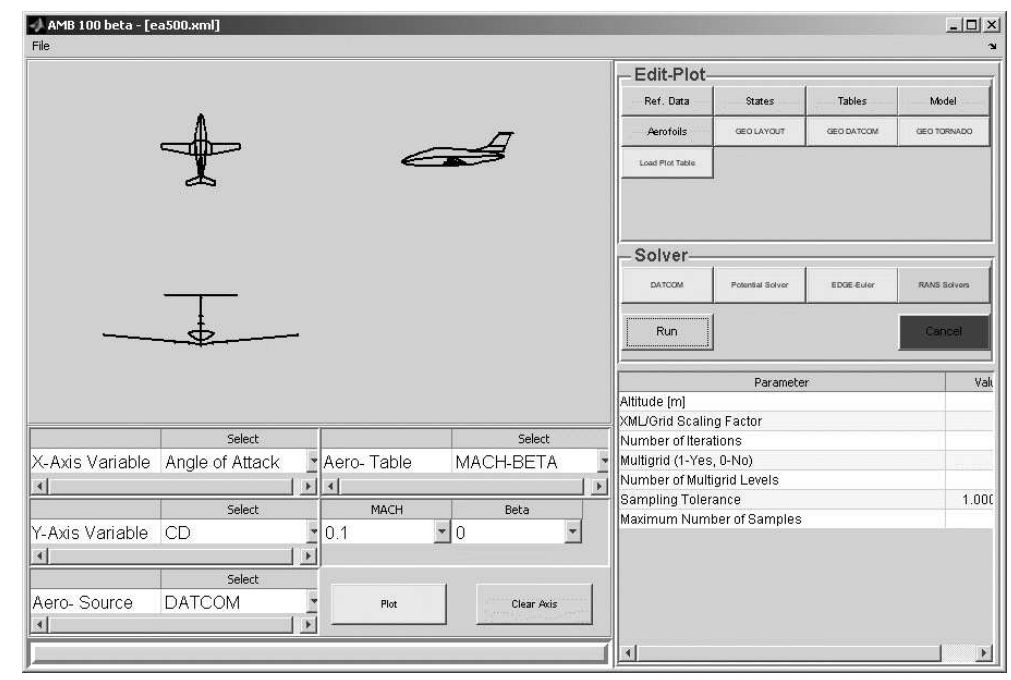

Figure 2. AMB GUI

\section{II.C. SCAA}

The Stability and Control Analyser and Assessor (SCAA) module of CEASIOM is used to assess the stability and control characteristics of the concept aircraft using the weights and balance data generated in AcBuilder and the aerodynamic data sets generated in AMB. This module allows the user to trim the aircraft at specified conditions, from which they can determine whether the resulting control surface deflections, angle of attack and drag are acceptable, or whether redesign of the aircraft is necessary. Figure 3 shows the GUI for SCAA.

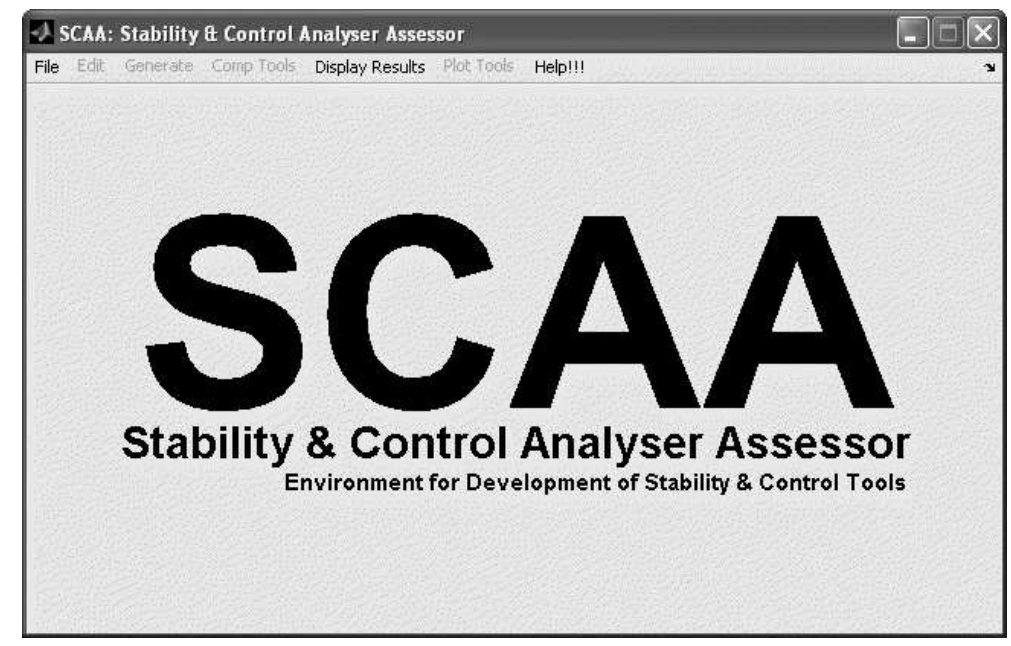

Figure 3. SCAA GUI

SCAA also linearises the aircraft model for each trim conditions, enabling an assessment of the characteristics of the dynamic modes to be made. Various specifications define requirements for the dynamic modes, such as the short period and Dutch Roll, of an aircraft which can vary depending on its intended role. SCAA can be used to determine the flight envelope in which these requirements are met, and also provides a tool for designing a feedback controller to move these modes if the requirements are not met. The feedback controller is tuned using an eigenstructure assignment algorithm. 


\section{Aircraft Configurations}

The purpose of this paper is to determine whether there are any drag benefits to using an asymmetric three-lifting-surface aircraft configuration, hereafter referred to as the Z-config. In order to carry out this investigation two aircraft configurations are needed: a baseline conventional configuration to provide a basis for comparison, and the Z-config itself. A description of these two models and their implementation in CEASIOM is given in the following sections.

\section{III.A. EA500}

The baseline aircraft selected for this investigation was the Eclipse Aerospace 500 Very Light Jet (EA500). It is a small business jet with up to 6 seats, a T-tail, a low wing and two fuselage mounted engines. According to its type certificate ${ }^{4}$ it has a maximum operating speed of Mach 0.65 and a maximum operating altitude of 41,000 ft. In order to create a model of the EA500 in CEASIOM a number of parameters that define the geometry of the aircraft needed to be determined. A detailed 3-view of the EA500 from the Eclipse Aerospace website ${ }^{3}$ was used to obtain these parameters. Values for some of the major dimensions are listed in Table 1.

\begin{tabular}{|l|c|c|}
\hline Dimension & Value & Units \\
\hline Fuselage Length & 9.2 & $\mathrm{~m}$ \\
\hline Wing Span & 11 & $\mathrm{~m}$ \\
\hline Wing Area & 13.92 & $\mathrm{~m}^{2}$ \\
\hline Mean Aerodynamic Chord & 1.385 & $\mathrm{~m}$ \\
\hline Horizontal Tail Span & 4.1 & $\mathrm{~m}$ \\
\hline Horizontal Tail Area & 3.24 & $\mathrm{~m}^{2}$ \\
\hline Vertical Tail Span & $\tilde{1} .7$ & $\mathrm{~m}$ \\
\hline Vertical tail Area & $\tilde{2} .8$ & $\mathrm{~m}^{2}$ \\
\hline
\end{tabular}

Table 1. EA500 dimensions

These dimensions were entered into the AcBuilder module of CEASIOM in order to generate a model of the EA500. The data from this model was then imported into SUMO in order to create a mesh for use by the Edge aerodynamic prediction code. The engines where removed from the model to aid in the aerodynamic calculations. The same will be done for the Z-config so this should have a minimal effect on the comparison of the two aircraft. SUMO was also used to specify the control surfaces of the aircraft as it has greater flexibility than AcBuilder in doing this. Figure 4 shows the model of the EA500 in SUMO.

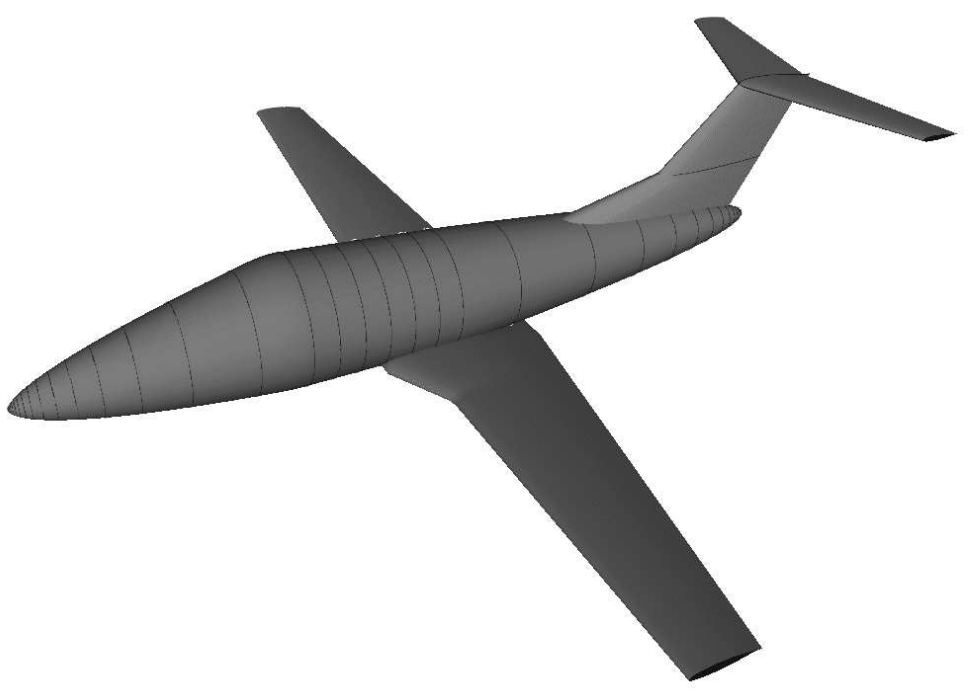

Figure 4. EA500 model in SUMO 
As the EA500 is an existing aircraft information on its weights and balance are available and therefore do not have to be predicted by CEASIOM. The EA500 type certificate ${ }^{4}$ contains details of the aircraft's maximum take-off weight (MTOW) and its acceptable Centre of Gravity (CG) range. Figure 5 shows the acceptable CG range of the EA500 for different weights. The datum from which the CG locations in this figure are measured is located 23.35 inches forward of the nose radome.

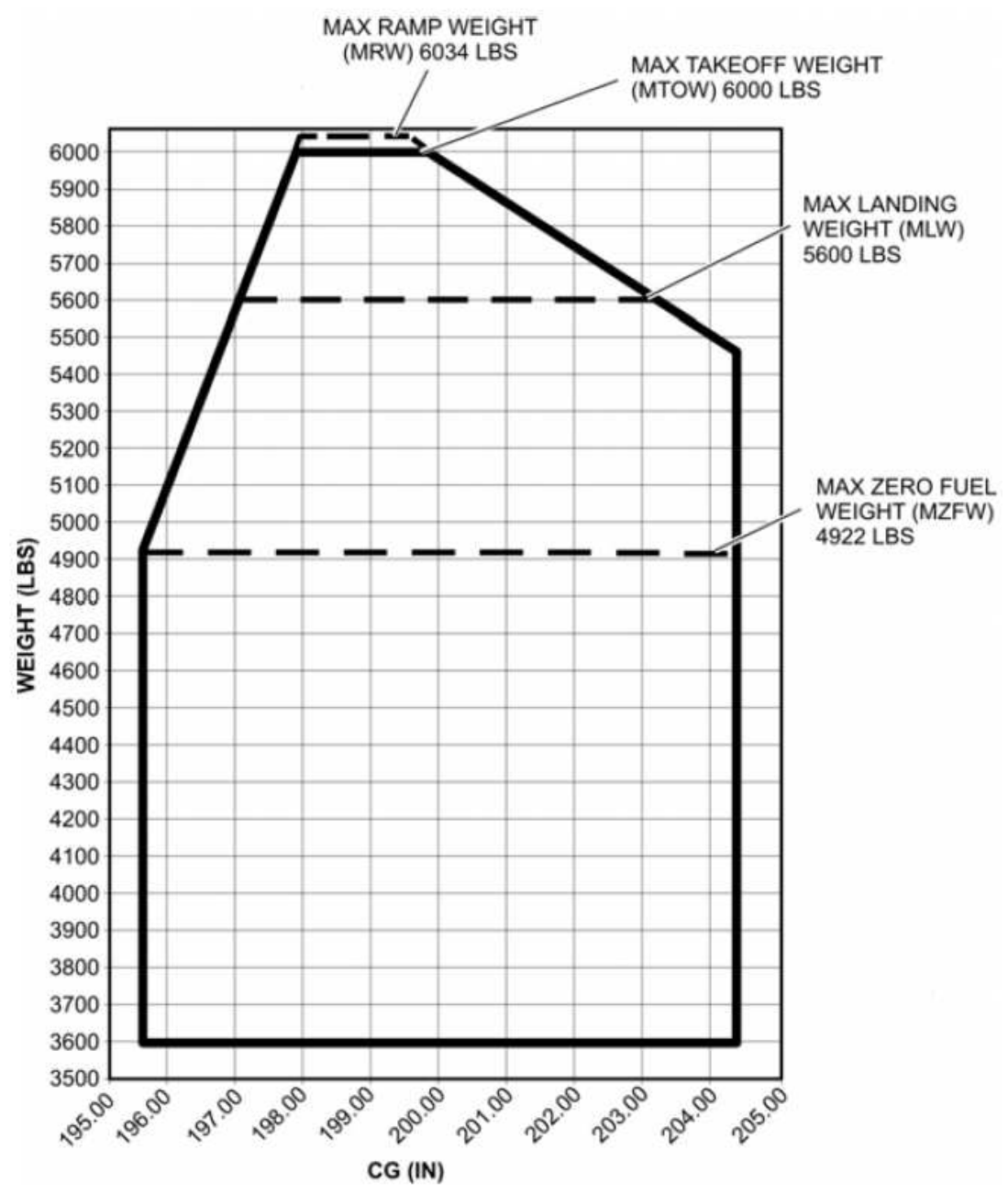

Figure 5. EA500 CG range

Table 2 shows the same data as Figure 5 but in metric units and with the CG locations measured from the nose radome tip.

\begin{tabular}{|l|c|c|c|}
\hline Condition & Mass (kg) & Fore CG Limit (m) & Aft CG Limit (m) \\
\hline Max Ramp Weight & 2734.7 & 4.438 & 4.473 \\
\hline Max Takeoff Weight & 2719.3 & 4.436 & 4.483 \\
\hline Max Landing Weight & 2540.1 & 4.415 & 4.6 \\
\hline Max Zero Fuel Weight & 2232.6 & 4.379 & 4.6 \\
\hline
\end{tabular}

The Empty Weight of the EA500 was not given in this document, however it was obtained from their website $^{3}$ as being $1648 \mathrm{~kg}$ (3634lbs). The fore and aft CG limits for masses between the Empty Weight and Max Zero Fuel Weight remain constant.

Before generating the aerodynamic tables, the point about which the aerodynamic coefficients are calculated needs to be selected. This would typically be the CG, but as the CG covers a range of values a 
single point still had to be chosen. The foremost acceptable CG for the EA500 at its MTOW was used, corresponding to a point $4.44 \mathrm{~m}$ aft of the aircraft nose.

The mesh generated using SUMO for the EA500 model was imported into the AMB module of CEASIOM where the Edge aerodynamic prediction code was used to generate aerodynamic data sets for the aircraft. These data sets consist of tables where the aerodynamic force and moment coefficients are calculated for a range of angles of attack, Mach numbers and one other variable. Four of these tables were generated with the other variable in each case being sideslip, elevator deflection, rudder deflection or aileron deflection. The range of values used for each of the variables in the tables is given in Table 3 .

\begin{tabular}{|c|c|c|}
\hline Variable & Values & Units \\
\hline AoA & {$\left[\begin{array}{llllllll}-4 & -2 & 0 & 2 & 4 & 6 & 8 & 10\end{array}\right]$} & $\circ$ \\
\hline Mach & {$\left[\begin{array}{lllll}0.2 & 0.35 & 0.5 & 0.65\end{array}\right]$} & - \\
\hline AoS & {$\left[\begin{array}{lllll}-6 & -3 & 0 & 3 & 6\end{array}\right]$} & $\circ$ \\
\hline Elevator & {$\left[\begin{array}{lllll}-15 & -7 & 0 & 7 & 15\end{array}\right]$} & $\circ$ \\
\hline Rudder & {$\left[\begin{array}{lllll}-20 & -10 & 0 & 10 & 20\end{array}\right]$} & o \\
\hline Aileron & {$\left[\begin{array}{lllll}-15 & -7 & 0 & 7 & 15\end{array}\right]$} & $\circ$ \\
\hline
\end{tabular}

Table 3. Variable values used for EA500 aerodynamic tables

Figures 6 and 7 show the lift and pitching moment coefficients for a range of mach numbers and angles of attack at the selected CG location.

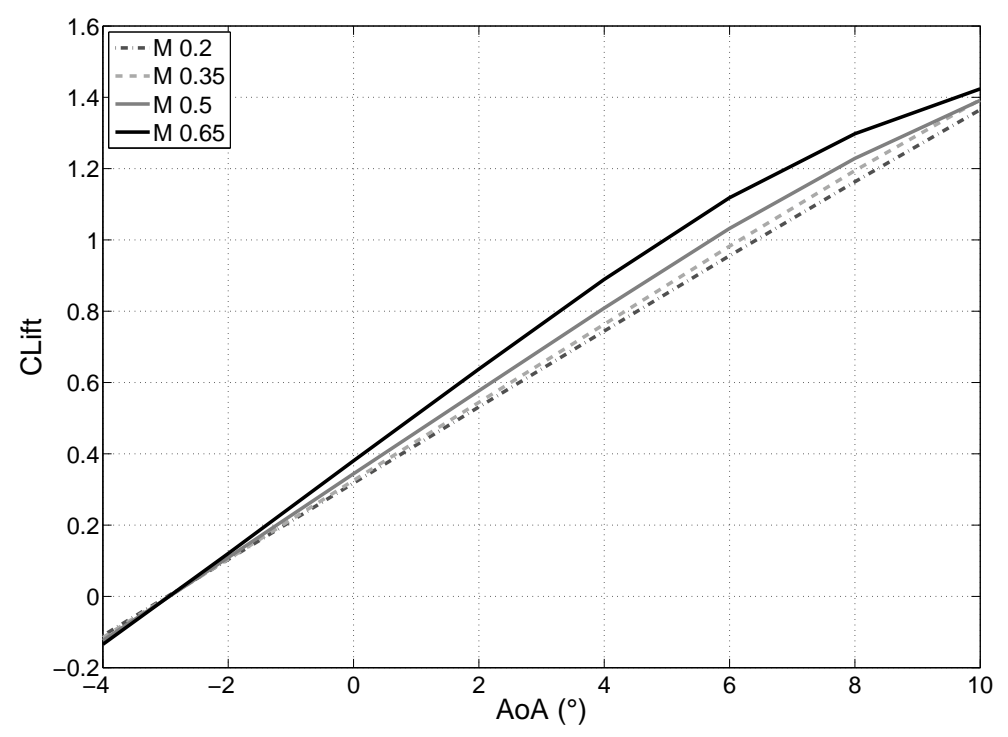

Figure 6. EA500 Lift Curve

It can be seen that both the lift-curve slope and pitching stiffness increase as the Mach number increases. The static margin as a fraction of the Mean Aerodynamic Chord (MAC) was calculated covering an angle of attack range of 0 to $2^{\circ}$ for each Mach number using the following equation:

$$
\text { Static Margin }=-\frac{\partial C_{m}}{\partial C_{L}}
$$

Figure 8 shows how the static margin of the EA500 varies with Mach number, going from $28.8 \%$ MAC at Mach 0.2 down to 25.5\% MAC at Mach 0.65. This puts the Neutral Point (NP) for the aircraft at 50.8\%MAC when travelling at Mach 0.65. The minimum static margin for the EA500 within its flight envelope can therefore be calculate to be $13.9 \% \mathrm{MAC}$ and so the aircraft is always statically stable longitudinally. 


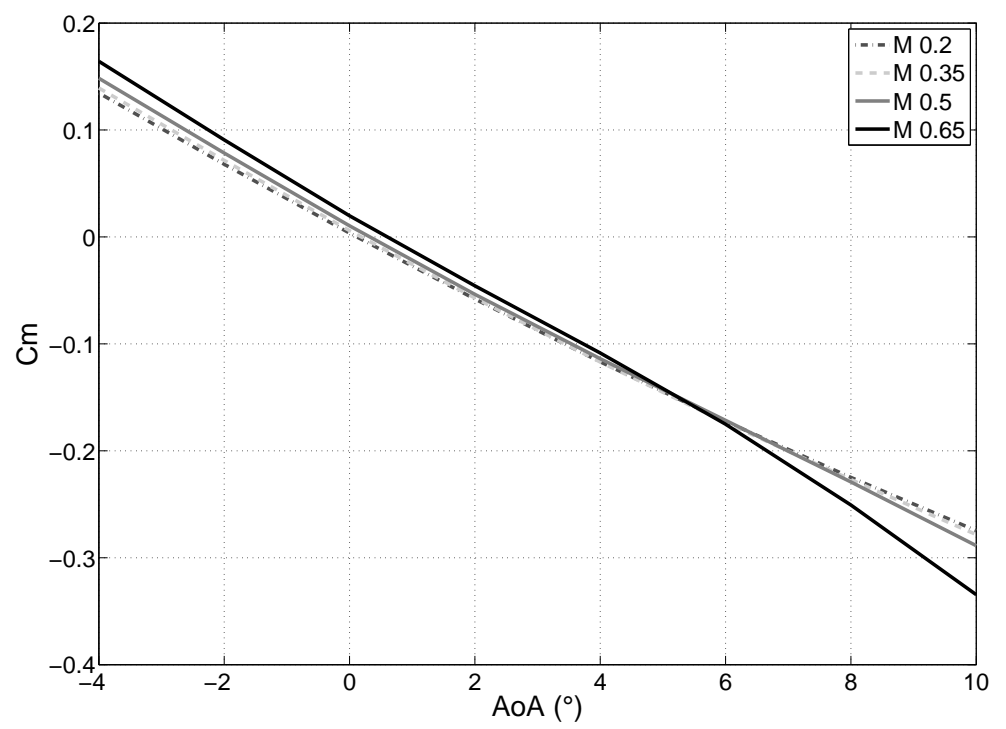

Figure 7. EA500 Pitching Moment Curve

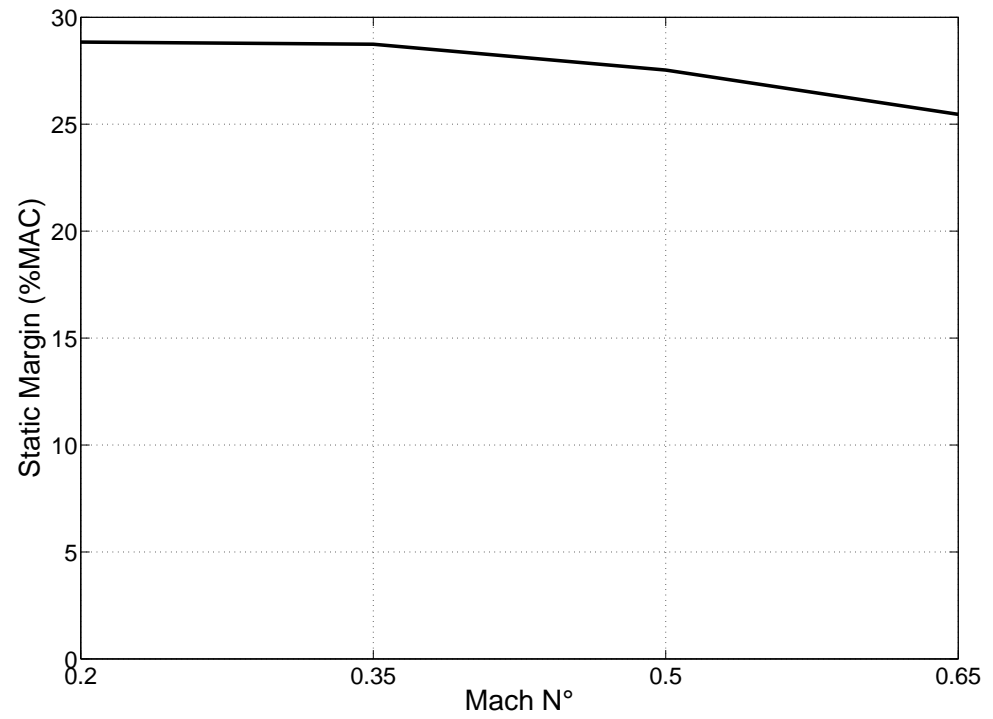

Figure 8. EA500 Static Margin 


\section{III.B. Z-config}

The Z-configuration is an asymmetric aircraft designed to fulfill the same role as the EA500. To ensure the comparison between the two aircraft is as useful as possible the EA500 fuselage was also used for the Z-config.

The Z-config has a split wing, with the starboard semi-wing located low on the fore fuselage, and the a port semi-wing located high on the aft fuselage. Both semi-wings have the same area and span however their sweep and dihedral differ. There is no horizontal tail, and the vertical tail is canted over to starboad to provide a third lifting surface. No engines were included on the Z-config model as the engines for the EA500 were removed for the aerodynamic calculations. Some of the major dimensions for the Z-config are listed in Table 4 .

\begin{tabular}{|l|c|c|}
\hline Dimension & Value & Units \\
\hline Fuselage Length & 9.2 & $\mathrm{~m}$ \\
\hline Starboard Semi-Wing Span & 6.17 & $\mathrm{~m}$ \\
\hline Starboard Semi-Wing Area & 5.95 & $\mathrm{~m}^{2}$ \\
\hline Port Semi-Wing Span & 6.17 & $\mathrm{~m}$ \\
\hline Port Semi-Wing Area & 5.95 & $\mathrm{~m}^{2}$ \\
\hline Canted Vertical Tail Span & 3.44 & $\mathrm{~m}$ \\
\hline Canted Vertical tail Area & 4.65 & $\mathrm{~m}^{2}$ \\
\hline \multicolumn{2}{|c|}{ Table 4. Z-config dimensions } \\
\hline
\end{tabular}

Figure 9 shows an overhead view of the two aircraft configurations from which the differences between them can clearly be seen.

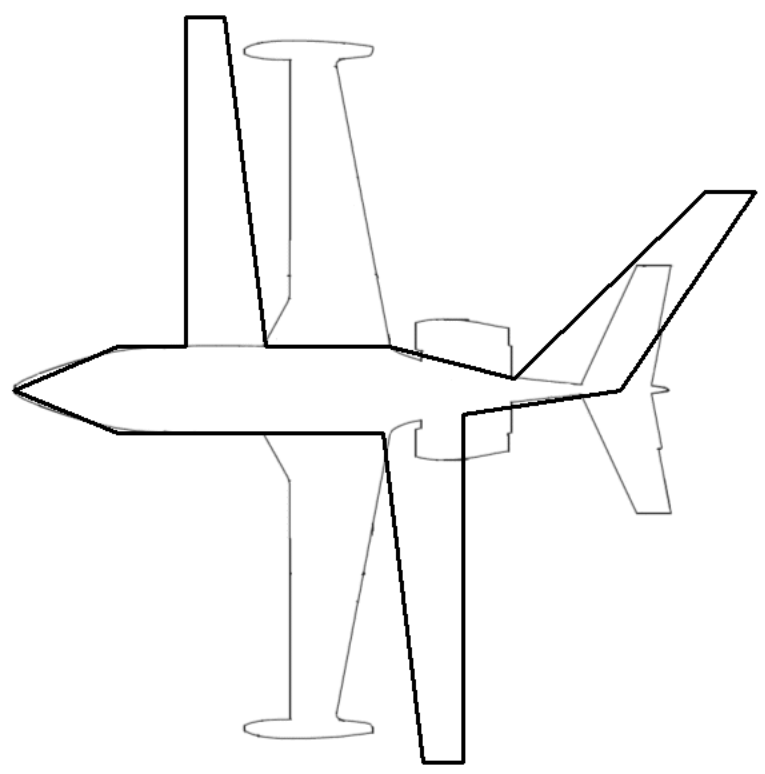

Figure 9. Overlay of EA500 and Z-config

The Z-config dimensions were entered into the AcBuilder module of CEASIOM in order to generate a model of the aircraft. This model was then imported into SUMO in order to create a mesh for use by the Edge aerodynamic prediction code. As with the EA500, no engines were included in the SUMO mesh of the Z-config. Figure 10 shows the model of the Z-config in SUMO.

Control surfaces were also added to the Z-config in SUMO. For this design five control surfaces were created, two ailerons on the outer sections of the semi-wings, two elevators on the inner sections of the semi-wings, and a rudder on the canted vertical tail.

As the Z-config is not an existing aircraft no data is available on its weights and balance. Whilst the 


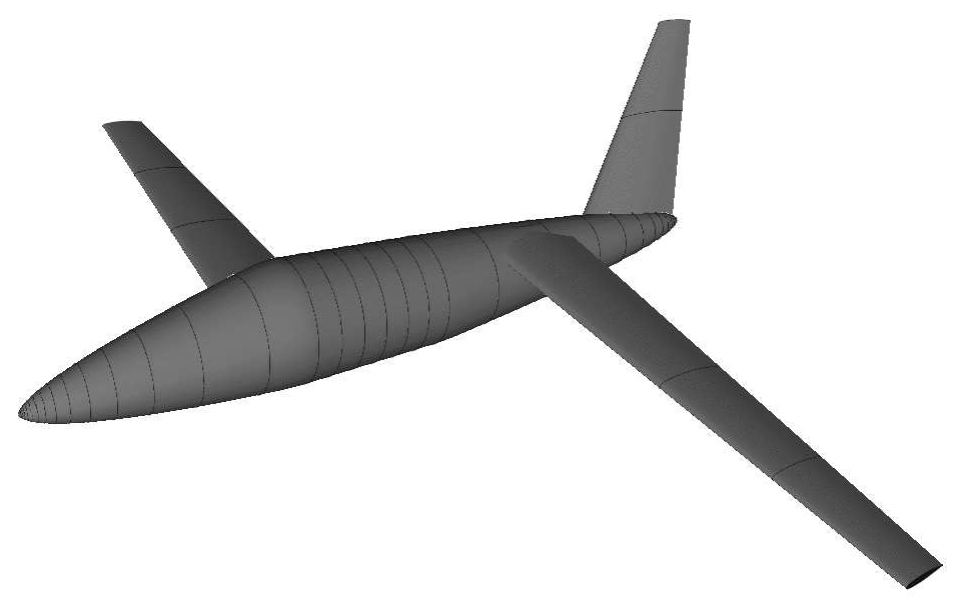

Figure 10. Z-config model in SUMO

AcBuilder module of CEASIOM is capable of estimating the aircraft's mass it was decided that the same mass data as for the EA500 would be used for the Z-config. This means that any drag savings achieved will be as a result of the configuration rather than a mass reduction. If the Z-config proves to be a viable aircraft then any mass changes and their effect on the aircraft's drag can be investigated at a later date.

Before generating the aerodynamic tables, the point about which the aerodynamic coefficients are calculated needs to be selected. The same point as used for the EA500 calculations was chosen, located $4.44 \mathrm{~m}$ aft of the aircraft nose.

The mesh generated using SUMO for the Z-config model was imported into the AMB module of CEASIOM where the Edge aerodynamic prediction code was used to generate aerodynamic data sets for the aircraft in the same way as for the EA500. Six tables were generated, one for sideslip and the other five for each of the control surfaces.

Figures 11 and 12 show the Z-Config's lift and pitching moment coefficients for a range of mach numbers and angles of attack at the selected CG location.

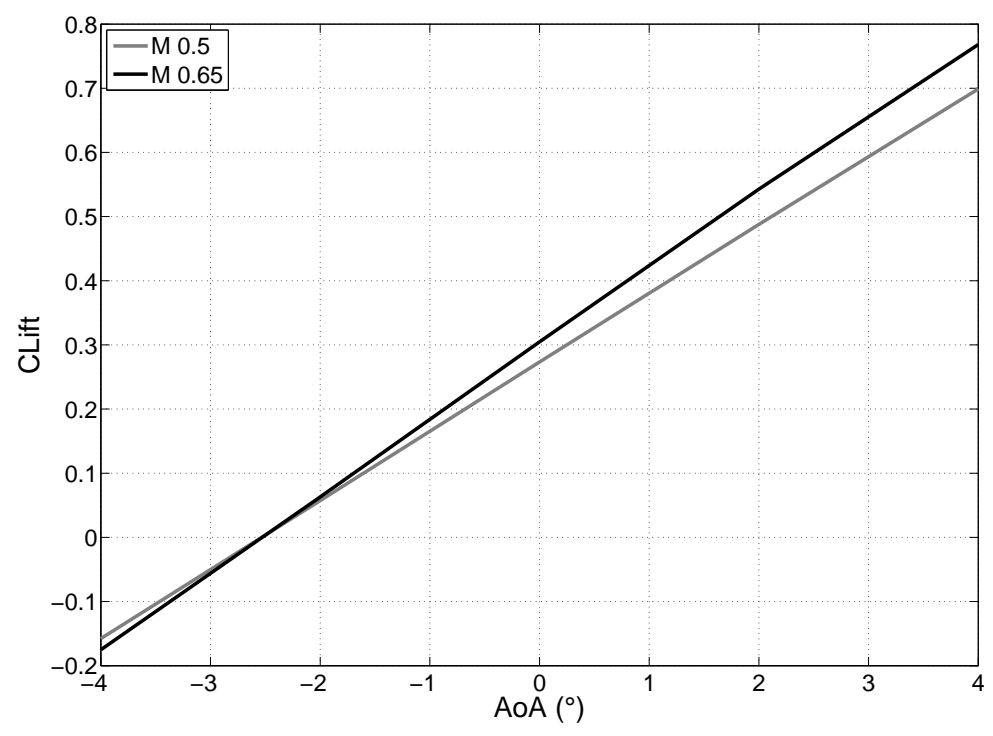

Figure 11. Z-Config Lift Curve

The static margin was calculated in the same way as for the EA500, and was found to be $29.6 \%$ for both Mach 0.5 and 0.65. This is up to $4.1 \%$ larger than for the EA500 at high speed, which will affect the trim 


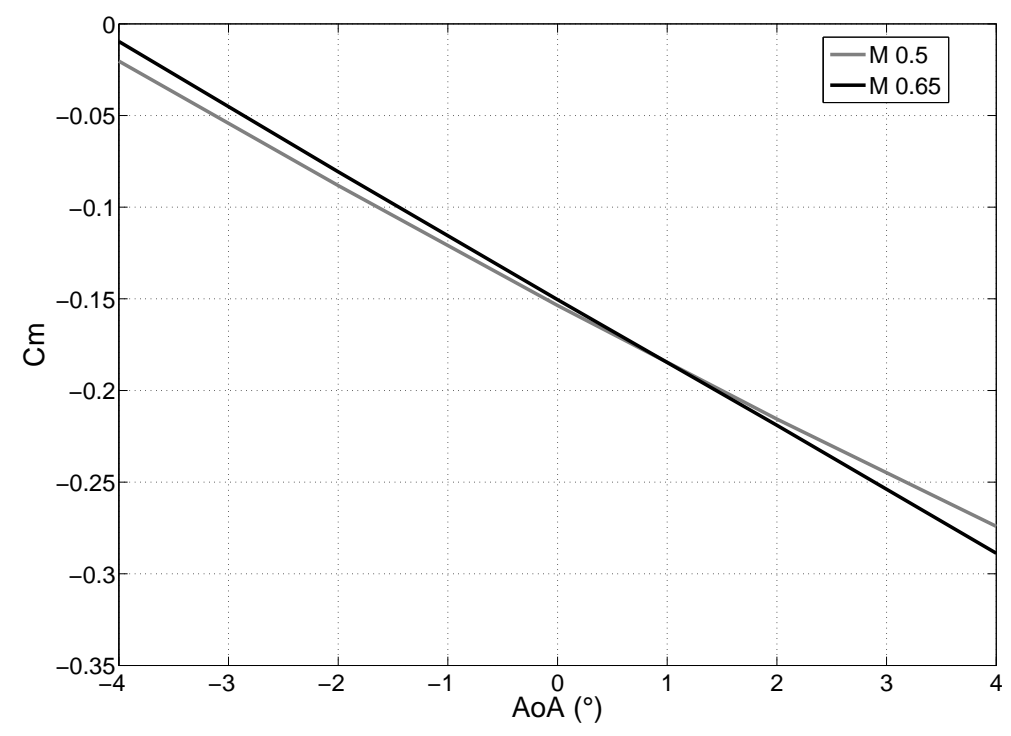

Figure 12. Z-Config Pitching Moment Curve

drag of the aircraft. In order to reduce the static margin on the Z-config to the same as that for the EA500 at cruise speed its CG has to be moved approximately $5.5 \mathrm{~cm}$ further aft. The SCAA software enables the user to move the $\mathrm{CG}$ and accounts for its effects when modelling the equations of motion of the aircraft.

\section{Trim Comparison}

In this section the two aircraft are trimmed in the cruise configuration using the SCAA module of CEASIOM. The trim values for the aircraft states, control surface deflections and thrust are then compared to assess whether the Z-config has the potential to be a more efficient aircraft than the EA500.

The flight condition selected for cruise was straight and level flight at Mach 0.65 and an altitude of 35,000 $\mathrm{ft}$. The masses for both aircraft were set to $2400 \mathrm{~kg}$ whilst their CGs were set to $4.44 \mathrm{~m}$ and $4.495 \mathrm{~m}$ from the nose for the EA500 and Z-config respectively.

When trimming the aircraft the forces and moments acting on it have to be balanced. The resulting moments all need to be zero as does the side force. The lift has to balance out the weight of the aircraft whilst the thrust has to balance out the drag.

\section{IV.A. EA500 Trim}

The EA500 was trimmed for the cruise flight conditions given previously. As the EA500 is a symmetric aircraft the lateral and directional force and moments will be balanced for zero deflections of the ailerons and rudder. The lift, drag and pitching moment of the aircraft can then be controlled by adjusting the angle of attack, elevator deflection and thrust. Table 5 gives these trim values for the cruise flight.

\begin{tabular}{|l|c|c|}
\hline Parameter & Value & Units \\
\hline Angle of attack & -1.16 & $\circ$ \\
\hline Elevator deflection & 1.25 & $\circ$ \\
\hline Thrust & 1.2 & $\mathrm{kN}$ \\
\hline
\end{tabular}

Table 5. EA500 cruise trim

The trim angle of attack is low in magnitude, which is likely to result in a low overall drag. The elevator deflection is also low in value, which will keep its contribution to the overall drag low. This all implies that the EA500 has been designed to keep drag low during cruise as would be expected. The thrust required to ahieve the trim was $1.2 \mathrm{kN}$, which can be compared to that for the Z-config at the same flight conditions. 


\section{IV.B. Z-Config Trim}

Unlike the EA500 the Z-config is asymmetric meaning that its lateral and directional forces and moments are non-zero for straight and level flight. There are up to eight parameters that can be varied to control the six forces and moments acting on the aircraft, and these are the angles of attack and sideslip, the five control surface deflections and the thrust. This means that there are two excess parameters than are needed to balance the forces and moments, which provides flexibility enabling several different trim configurations to be explored.

Trim configurations using the five control surfaces and engine thrust to trim the aircraft whilst the sideslip was kept at zero were analysed. Several different angles of attack were used in these configurations, with the smallest control surface deflections achieved when the angle of attack was three degrees. The control surface deflections required to achieve trim are given in Table 6.

\begin{tabular}{|l|c|c|}
\hline Control & Value & Units \\
\hline Left elevator deflection & -9.4 & ${ }^{\circ}$ \\
\hline Right elevator deflection & -11.5 & $\circ$ \\
\hline Left aileron deflection & 8.3 & $\circ$ \\
\hline Right aileron deflection & 3.3 & $\circ$ \\
\hline Rudder deflection & -8.9 & $\circ$ \\
\hline Thrust & 1.4 & $\mathrm{kN}$ \\
\hline
\end{tabular}

Table 6. Z-config control effector values required for trim

As can be seen the required thrust to trim the Z-config is slightly larger than that required to trim the EA500. One likely reason for this is that there is an increase in drag resulting from the significantly larger control surface deflections required for trim.

In order to determine why the control surface deflections required for trim are so much larger for the Z-config than the EA500, the effectiveness of each control surface is analysed. In order to trim the Z-config good control authority in all channels is needed. The impact that deflections of each of the control surfaces has on the aerodynamic moment coefficients and the lift and side forces were calculated and normalised to determine what channels they have the greatest effect on. Table 7 gives the relative impact that each control effector has on each of the force and moment coefficients.

\begin{tabular}{|l|c|c|c|c|c|}
\hline Control & \multicolumn{5}{|c|}{ Normalised Effect of Deflection on: } \\
\hline Surface & $C_{L}$ & $C_{Y}$ & $C_{l}$ & $C_{m}$ & $C_{n}$ \\
\hline Left elevator & 0.53 & -0.07 & -0.10 & -0.84 & -0.02 \\
\hline Right elevator & 0.56 & 0.13 & 0.08 & 0.81 & 0.03 \\
\hline Left aileron & 0.53 & -0.06 & -0.21 & -0.82 & -0.01 \\
\hline Right aileron & 0.65 & -0.03 & 0.23 & 0.72 & 0.01 \\
\hline Rudder & 0.24 & -0.17 & 0.05 & -0.95 & -0.08 \\
\hline
\end{tabular}

Table 7. Control authority of each Z-config control surface

From this table it can be seen that there is good control authority in the longitudinal channels of lift and pitch, but control authority in the lateral and directional channels is less strong. The ailerons have the greatest effect on the rolling moment as to be expected, but because the tail is canted over the rudder does not have a significant effect on the yawing moment coefficient, instead it primarily affects the pitching moment coefficient. In contrast, deflecting the rudder on the EA500 affects its yawing moment coefficient over 10 times greater than the pitching moment coefficient.

This analyses shows that a significant problem with the Z-config design is the lack of effective yaw control which, coupled with its asymmetry, means that there is a trim drag penalty during cruise preventing it from being more efficient than the EA500. If this concept is to be explored further then the problem with directional control authority needs to be addressed. 


\section{Conclusions}

A novel asymmetric aircraft configuration was suggested as offering possible efficiency benefits when compared to a more conventional design. The CEASIOM environment was used to assess both the original conventional aircraft and this novel design to determine whether the potential efficiency improvements can be achieved in cruise. Aerodynamic data sets for both aircraft were generated after which they were trimmed for cruise from which the thrust required for this trim was obtained. A higher thrust was required for the novel configuration than the conventional meaning that the potential efficiency improvements were not realised. The reason for this appears to be the large control surface desflections required for trim due to the poor directional control authority. If this design is to be explored further then this problem needs to be addressed.

This study has demonstrated the usefulness of the CEASIOM software environment, which enables novel designs such as the Z-config to be analysed and their potential assessed. Whilst the Z-config design has not proved suitable it was possible not only to demonstrate this but to determine a factor that likely contributed to its failure to achieve the potential efficiency improvements. This provides the aircraft designer with useful knowledge and feedback if the design is to be reworked at a later stage.

\section{References}

${ }^{1}$ von Kaenel, R., Rizzi, A., Oppelstrup, J., Goetzendorf-Grabowski, T., Ghoreyshi, M., Cavagna, L., Berard, A., "CEASIOM: Simulating Stability \& Control With CFD/CSM in Aircraft Conceptual Design", Paper 061, 26th ICAS, Alaska, Sept 2008.

2 www.ceasiom.com

${ }^{3}$ www.eclipseaerospace.net, January 2010

${ }^{4}$ Eclipse Aerospace, EA500 "Type Certificate Data Sheet No. A00002AC", Department of Transportation, Federal Aviation Administration, Revision 4, September 2009. 